\title{
Comment on "The changing face of paracetamol toxicity and new regimens for an old antidote acetylcysteine"
}

\author{
Alaa Daoud ${ }^{1}$, Kim Dalhoff ${ }^{2}$, and Tonny Petersen ${ }^{2}$ \\ ${ }^{1}$ Department of Clinical Pharmacology and The Danish Poison Information Centre, \\ Bispebjerg and Frederiksberg Hospital, Copenhagen University Hospital, Copenhagen, \\ Denmark \\ ${ }^{2}$ Bispebjerg and Frederiksberg University Hospital
}

September 28, 2020

\section{Comment on "The changing face of paracetamol toxicity and new regimens for an old antidote acetylcysteine"}

Dear Editor,

We have with interest read the recently published commentary by Isbister and Chiew ${ }^{1}$ in which current approaches for the treatment of paracetamol (PCM) poisoning with $\mathrm{N}$-acetylcysteine (NAC) were described. While we appreciate the authors' valuable comments on the challenges in managing this common poisoning, we noticed the omission of the Danish NAC regimen.

NAC has been established as a highly efficient antidote in preventing PCM-induced hepatotoxicity when administered within $8 \mathrm{~h}$ of PCM ingestion. ${ }^{2}$ Treatment delays because of pending laboratory results do not harmfully affect the outcome if NAC is administered within $8 \mathrm{~h}$. However, it is frequent occurring that patients get treated beyond that time in e.g. the UK, ${ }^{3}$ thus potentially decreasing the efficacy of NAC.

In Denmark a 20-hour two-bag regime has been used for more than seven years. ${ }^{4}$ All patients suspected of poisoning with more than $6 \mathrm{~g}$ PCM are treated with NAC without risk stratification according to a nomogram. Patients deliberately poisoning themselves with PCM may not report reliable information of the time of ingestion and dose of PCM. If a nomogram is used without an accurate time of ingestion, the estimation of the risk of hepatotoxicity is unreliable. It is well documented that patients have been withheld treatment with NAC due to misinterpretation of the nomogram resulting in hepatotoxicity. ${ }^{5}$ Concomitant overdosing of several drugs that delay the time to peak PCM concentration is common and may result in crossing from below to above the treatment line ${ }^{5}$ leaving line crossers who required treatment untreated. The same is relevant in cases of massive PCM overdosing alone (drug bezoar) or poisonings with extended-release PCM formulations (late PCM peak). ${ }^{6}$ Furthermore, it is well-known that the half-life of PCM in liver injury exceeds the expected $4 \mathrm{~h}$ used for treatment decision in the nomogram, thus further questioning the reliability of the nomogram as a risk stratification tool for patients suffering from liver diseases. Although the Danish regime further reduces the risk of hepatoxicity, because of incorrectly withheld or delayed NAC treatment compared to other regimes based on a nomogram, it comes at a cost of more patients being treated.

We believe that all approaches deserve mention in order to identify the most effective and safe approach to this poisoning. Therefore, we should consider the effectiveness, duration and safety of choice of treatment including the incidence of anaphylactoid reactions to NAC while assuring that nobody is wrongly withhold NAC treatment. Not to mention, that the abovementioned approach is associated with a comparable incidence of anaphylactoid reactions when compared to other approaches. ${ }^{4,7}$ It is worth mentioning that the primary factor limiting a faster delivery of the antidote is the development of anaphylactoid reactions. It 
is to be shown if pre-administrations of antihistamines can reduce the dose-dependent side-effect leading to development of faster NAC regimes ${ }^{7}$.

We declare no competing interests.

Alaa Daoud (orcid: 0000-0002-8714-4028) a,b $^{\mathrm{a}}$, Kim Peder Dalhoff ${ }^{\mathrm{a}, \mathrm{b}}$, Tonny Studsgaard Petersen (orcid: 0000-0002-9974-2738) $)^{\mathrm{a}, \mathrm{b}}$

${ }^{a}$ Department of Clinical Medicine, Faculty of Health and Medical science, Copenhagen University, Copenhagen, Denmark

${ }^{\mathrm{b}}$ Department of Clinical Pharmacology and The Danish Poison Information Centre, Bispebjerg and Frederiksberg Hospital, Copenhagen University Hospital, Copenhagen, Denmark

*Correspondence to Alaa Daoud, Alaa.Ahmed.Daoud@regionh.dk

1. Isbister GK, Chiew A. The changing face of paracetamol toxicity and new regimens for an old antidote acetylcysteine. British Journal of Clinical Pharmacology . n/a(n/a). doi:10.1111/bcp.14495

2. Prescott LF, Illingworth RN, Critchley JA, Stewart MJ, Adam RD, Proudfoot AT. Intravenous Nacetylcystine: the treatment of choice for paracetamol poisoning. $\operatorname{Br}$ Med J . 1979;2(6198):1097-1100. doi:10.1136/bmj.2.6198.1097

3. The College of Emergency Medicine. Paracetamol Overdose Clinical Audit 2013-14. Accessed September 7, $2020 . \quad$ https://www.rcem.ac.uk/docs/Previous\%20Audits/CEM8120Paracetamol\%20Overdose\%20national\%20report.pdf

4. Daoud A, Dalhoff KP, Christensen MB, Bøgevig S, Petersen TS. Two-bag intravenous Nacetylcysteine, antihistamine pretreatment and high plasma paracetamol levels are associated with a lower incidence of anaphylactoid reactions to N-acetylcysteine. Clinical Toxicology . 2020;58(7):698-704. doi: $10.1080 / 15563650.2019 .1675886$

5. Mutsaers A, Green JP, Sivilotti MLA, et al. Changing nomogram risk zone classification with serial testing after acute acetaminophen overdose: a retrospective database analysis. Clinical Toxicology . 2019;57(6):380386. doi:10.1080/15563650.2018.1529320

6. Bizovi KE, Aks SE, Paloucek F, Gross R, Keys N, Rivas J. Late Increase in Acetaminophen Concentration After Overdose of Tylenol Extended Relief. Annals of Emergency Medicine . 1996;28(5):549-551. doi:10.1016/S0196-0644(96)70119-1

7. Mullins ME, Yu M, O'Grady L, Khan S, Schwarz ES. Adverse reactions in patients treated with the onebag method of N-acetylcysteine for acetaminophen ingestion. Toxicology Communications . 2020;4(1):49-54. doi: $10.1080 / 24734306.2020 .1770498$ 